\title{
Family planning in genitourinary medicine: an opportunistic service?
}

\author{
L Masters, H Nicholas, P Bunting, J Welch
}

\begin{abstract}
Objective-To evaluate and serve the need for contraception in those not using conventional sources of family planning services.

Setting-South London outpatient genitourinary medicine department at King's College Hospital.

Design and subjects-Prospective study of 200 women seen consecutively in the clinic by the same doctor (LM) during 1993. Women at risk of unwanted pregnancy were identified, and offered immediate contraceptive provision or referral. Results-15 women $(7 \cdot 5 \%)$ were using no contraception, despite being sexually active and not wishing to conceive; of these two presented with an unwanted pregnancy. A further 23 women $(11 \cdot 5 \%)$ were not using their chosen contraception effectively, and another 20 women were unclear about contraceptive methods and wanted advice. Young women were most at risk; $14 \%$ of those aged 25 years and under were using no contraception. Eight women wished to defer contraceptive advice; of these four defaulted from follow up. Eighteen women $(9 \%)$ wanted immediate contraceptive supplies. Ten of 18 returned a follow up questionnaire; all these women were satisfied with the contraceptive advice service received.

Conclusions-Absent or ineffective contraception is common in women attending an inner city genitourinary medicine clinic. Immediate provision of contraceptive education, advice and supplies is welcomed by patients.
\end{abstract}

(Genitourin Med 1995;71:103-105)

Keywords: Family planning, Genitourinary medicine.

\section{Introduction}

The Health of the Nation ${ }^{1}$ white paper states amongst its objectives a need to ensure effective family planning services for those people who want them, and to reduce the rate of unwanted pregnancies. The specific aim is to halve the pregnancy rate in the under 16 age group by the year 2000. This is proving hardest to achieve in areas which are socioeconomically deprived. ${ }^{2}$ The Department of Genitourinary (GU) Medicine at King's College Hospital sees 19000 women a year. Fifty six per cent of our population are socioeconomically deprived. ${ }^{3}$ Conception rates exceed the national average for all age groups, and the termination of pregnancy (TOP) to live birth ratio is one of the highest in the country. ${ }^{4}$ We assessed the need for an "opportunistic" family planning service within the GU clinic, in which advice was offered to those most at risk of unwanted pregnancy, and follow up was arranged locally to make most economical use of existing services.

\section{Methods}

A structured interview was held with 200 women seen consecutively in the clinic by the same doctor (LM). Demographic information and details of sexual history and contraceptive practices were recorded. Compliance with contraception was assessed at interview and was defined as "good" (over $90 \%$ compliance), "partial" (70-90\%) or "poor" (less than $70 \%$ ).

A group of women at high risk of unwanted pregnancy was identified. They were those who were sexually active, without contraception, and who did not want to conceive. Contraceptive advice, supplies or referral were offered by the doctor where appropriate, especially targeting this "high risk" group. Advice and techniques were reinforced by a nurse with family planning training. Follow-up appointments with a general practitioner (GP) or family planning clinic were facilitated. Women given a new method of contraception were also asked to fill in a short feed-back questionnaire. Results from the structured interview were analysed using EPI. INFO and STATCALC programmes using chi square, $p$ for significance at 0.05 level and odds ratios with $95 \%$ confidence intervals.

\section{Results}

Of 200 women seen, $94 \quad(47 \%)$ were Caucasian, 79 (39.5\%) black Caribbean, nine $(4.5 \%)$ black African and $18(9 \%)$ were of other origins. The majority were aged 30 years or under (table 1) and sexually active at

\section{Table 1 Ages of participants}

\begin{tabular}{lcc}
\hline Age (years) & Frequency & $\%$ \\
\hline Under 16 & 3 & $1 \cdot 5$ \\
$16-20$ & 32 & 16 \\
$21-25$ & 54 & 27 \\
$26-30$ & 65 & $32 \cdot 5$ \\
$31-35$ & 21 & $10 \cdot 5$ \\
$36-40$ & 12 & 6 \\
Over 40 & 13 & $6 \cdot 5$ \\
Total & 200 & 100 \\
\hline
\end{tabular}


Table 2 Current sexual activity

\begin{tabular}{lcc}
\hline & Frequency & $\%$ \\
\hline Not sexually active & 26 & 13 \\
Trying to conceive & 26 & 13 \\
Using contraception & 128 & 64 \\
Proven infertile & 4 & 2 \\
At risk of unwanted pregnancy & 15 & $7 \cdot 5$ \\
Female partner only & 1 & $0 \cdot 5$ \\
Total & 200 & 100 \\
\hline
\end{tabular}

Table 3 Obstetric history

\begin{tabular}{lcc}
\hline & Frequency & $\%$ \\
\hline Never pregnant & 69 & $34 \cdot 5$ \\
Nulliparous with past miscarriage & 8 & 4 \\
Nulliparous with past TOP & 37 & $18 \cdot 5$ \\
$\quad$ including TOP and miscarriage) & & \\
Parous without TOP & 50 & 25 \\
Parous with TOP & 36 & 18 \\
Total & 200 & 100 \\
\hline
\end{tabular}

the time of interview (table 2). Fifteen women $(7 \cdot 5 \%)$ were classed as "high risk". Two of these were attending with an unwanted pregnancy. Twelve $(80 \%)$ of the high risk group were under 25 years old $(p=0.019$, odds ratio $5 \cdot 02$, confidence limits $1 \cdot 25$ to $28 \cdot 76$ ).

Ten percent of the women said they had not been sexually active during the last three months, $73 \%$ said they had been in monogamous relationships, $12.5 \%$ said they had had two or more regular partners and $4.5 \%$ had had casual partners only. Seventy-three women $(36.5 \%)$ had had at least one termination (table 3 ). Forty-five $(22.5 \%)$ said that they did not want any more children.

Choice of contraception and compliance rates are shown in table 4 . Twenty-three women (18\% of those using contraception) were not using their chosen contraception effectively in addition to the 15 who were completely unprotected. There were no significant differences in either choice of contraception or compliance between ethnic groups. Most of those using contraception attended their GPs $(45 \%)$ or family planning clinics (23\%) for advice, but the pharmacist was the most popular source of condoms (16\%). Nine $(4 \cdot 5 \%)$ were not registered with a GP. Fiftyeight women $(29 \%)$ were unclear about contraceptive methods and needed advice, including the entire at risk group who were

Table 4 Choice of contraception and compliance

\begin{tabular}{lccc}
\hline & Frequency & Compliance & \\
\cline { 2 - 4 } & No (\%) & Good & Partial/Poor \\
\hline No contraception/ & $72(36)$ & - & - \\
$\quad$ not sexually active & $62(31)$ & 56 & 6 \\
$\begin{array}{l}\text { Combined oral } \\
\quad \text { contraceptive pill (OCP) }\end{array}$ & $7(3 \cdot 5)$ & 4 & 3 \\
Progesterone only pill (POP) & $27(13 \cdot 5)$ & 17 & 10 \\
Condom (male or female) & $3(1 \cdot 5)$ & 2 & 0 \\
Cap & $6(3)$ & 6 & 0 \\
Intra-uterine contraceptive & $11(5 \cdot 5)$ & 11 & 1 \\
$\quad$ device (IUCD) & $4(2)$ & 3 & 0 \\
Sterilisation & $2(1)$ & 0 & 23 (18\%) \\
Natural/withdrawal & $6(3)$ & 6 & \\
Spermicide and condom & $200(100 \%)$ & $105(82 \%)$ & \\
Injectable progestogen & & & \\
Total & & & \\
\hline
\end{tabular}

Table 5 Contraception provided at clinic consultations

\begin{tabular}{lc}
\hline & Frequency \\
\hline No contraception given & 176 \\
Condoms & $8^{\star}$ \\
OCP/POP & 3 \\
Injectable progestogen & 4 \\
Cap & 1 \\
Female condom & 3 \\
Spermicides & 2 \\
Referred elsewheret & 3 \\
Total & 200 \\
\hline
\end{tabular}

«Not including condoms given purely for safer sex in the presence of other contraception

†Referrals to ante-natal clinic or to family planning clinic/GP for IUCD insertion

using no contraception at all. Eighteen patients $(9 \%)$ wanted contraceptive supplies at the first appointment. Eight preferred to wait until a follow up visit, but four then failed to return and three subsequently declined further contraceptive advice. Table 5 shows the range of contraception provided at these consultations.

Ten of the eighteen women (56\%) given a new method of contraception returned the follow up questionnaire, and all these were satisfied with the family planning service they had received at the $\mathrm{GU}$ clinic.

\section{Discussion}

Our findings confirm other studies ${ }^{5}$ in finding that absent or ineffective contraception is common in GUM clinic attenders. Young women are most at risk of unwanted pregnancy, with $14 \%$ of those aged 25 and under using no contraception. The need for patient education is great, with $29 \%$ of women feeling unclear about contraceptive methods. Poor compliance leading to ineffective contraception was widespread: inadequate patient education was the major cause.

Such a substantial need for advice about contraception, and low attendance rates for specialist clinics, ${ }^{6}$ suggest that family planning expertise needs to be available in routine GU clinics as well as in designated family planning sessions. Such expertise can be provided in a variety of ways, by both doctors and nurses. Doctors in GU medicine training posts can readily also undertake the training necessary for the diploma in family planning, as can doctors in staff grade posts. Clinical assistants with a family planning background can continue to work in both fields, to the benefit of each specialty. Nurses with family planning training are well placed to educate and advise patients about their contraception, and, in our experience, value this extension of their roles. Health advisers can also make a major contribution by discussing contraception in addition to other aspects of sexual health.

Constraints on time may make it unfeasible to carry out some procedures, such as fitting a cap or a coil, in a busy GU medicine clinic, unless additional staff can be funded and made available. Initiating hormonal contraception for women at high risk of unwanted pregnancy is generally easier to achieve, and can be facilitated by enabling the nursing staff 
to receive family planning training, so that they can provide most of the patient education required. Advising women who are using their chosen method of contraception ineffectively takes relatively little medical and nursing time, but is an important component of sexual health promotion.

Where appropriate we recommend giving family planning advice to patients at high risk of unwanted pregnancy, and initiating contraception, at the first visit, as our study showed little chance of a second opportunity. The women can then attend their GP or local family planning clinic for follow up but may need information about available services and help in making appointments. This approach was welcomed by substantial numbers of our patients, including over half those at greatest risk of unwanted pregnancy.

We feel that, especially in deprived areas, opportunistic family planning could make useful contribution to reducing unwanted pregnancy in young women.

1 Secretary of State for Health. "The health of the nation: a strategy for

2 Smith T. Influence of socio-economic factors on attaining targets for reducing teenage pregnancies. BMF 1994, 306:1232-5.

3 South East London Commissioning Agency. The Health of South East Londoners. Annual Public Health Report 1991: Socio-economic characteristics of the SELCA population. London: Camberwell Health Authority 1992:8-10.

4 South East London Commissioning Agency. Gainin Health. Annual report of the Director of Public Health 1992. Chapter 2: Profile of the population of the SELCA area, pp 11-17; Chapter 6: Sexual Health, pp 26-30. London: South East London Commissioning Agency 1993.

5 Queen HF, Ward H, Smith C, Woodroffe C. Women's Health: Potential of better co-ordination of services. Genitourin Med 1991;67:215-9.

6 Tobin JF, Roy RB. Are we failing our teenagers? Value of a family planning service for teenagers within a sexually family planning service for teenagers within a 